\section{Conhecimento sobre anticoncepcionais em uma população de 15 anos ou mais de uma cidade do Sul do Brasil}

\author{
Knowledge about contraceptives in a population \\ 15 years or older in a southern Brazilian city
}

\section{Abstract}

In Brazil, prevalence of contraceptive use is high, but incorrect and inappropriate use is frequent, suggesting limited knowledge about methods. This study thus evaluated the knowledge about the most widely used contraceptive methods, measured through a score (0-10) and related factors. A population-based cross-sectional study was performed, with 3,542 subjects ages 15 or older, residing in the urban area of Pelotas, Rio Grande do Sul State. The mean overall knowledge score was $4.65(s d=2.07)$ : $5.02(s d=2.10)$ for women and $4.18(s d=1.92)$ for men. Lower age, higher schooling, a report of unwanted pregnancy, and any lifetime use of contraceptive methods (exclusive or combined) were associated with higher knowledge scores among men, while for women, the determinants of higher knowledge were higher age, living with a partner, higher schooling, higher socioeconomic status, not reporting a religion, and any lifetime use of contraceptive methods (exclusive or combined). Despite high prevalence of any lifetime contraceptive use (75.3\%), knowledge is still limited about the most widely used methods, as well as about the menstrual cycle and fertile period.

Health Knowledge, Attitudes, Practice; Contraception; Contraceptive Agents
Vera Maria Vieira Paniz 1 Anaclaudia Gastal Fassa 1 Marcelo Cozzensa da Silva 1

\section{Introdução}

O conhecimento sobre métodos anticoncepcionais pode contribuir para que os indivíduos escolham o método mais adequado ao seu comportamento sexual e às suas condições de saúde, bem como, utilizem o método escolhido de forma correta. Assim, esse conhecimento deve estar relacionado à prevenção da gravidez indesejada, do aborto provocado, da mortalidade materna e de outros agravos à saúde relacionados à morbi-mortalidade reprodutiva 1 . Promover o acesso a tais informações e aos meios para a regulação da fecundidade é um dos aspectos importantes do planejamento familiar, uma das ações do Programa de Assistência Integral à Saúde da Mulher - PAISM, implantado em 19862.

Embora os métodos mais conhecidos sejam o anticoncepcional oral, preservativo masculino, esterilização feminina, DIU e abstinência periódica, a contracepção se restringe geralmente ao uso do anticoncepcional oral e da esterilização feminina ${ }^{3}$. Para Monteiro 4, esse panorama evidencia a precariedade da atenção à mulher, que adota procedimentos irreversíveis ante o estreito leque de opções e acesso restrito à informação, especialmente para aquelas que dependem do Sistema Único de Saúde (SUS). Desse nodo, a diversidade de métodos contraceptivos contrasta com a dificuldade no acesso e limitada informação sobre a ampla va- 
riedade de métodos anticoncepcionais existentes 3 , indicando um descompasso entre o que é proposto pelo programa de planejamento familiar e aquilo que é efetivamente implementado.

Pesquisa nacional realizada em 1996 revela que a utilização de algum método anticoncepcional entre mulheres em idade fértil foi de $76,7 \% 1$. Considerando-se todas as mulheres sexualmente ativas nesta pesquisa, o uso de algum método anticoncepcional foi de $55,4 \%$ : $27,3 \%$ estavam esterilizadas, $15,8 \%$ usavam anticoncepcional oral, $4,3 \%$ usavam preservativo masculino e 8,0\% outros métodos (injetáveis, DIU, vasectomia e métodos naturais). Em Pelotas, Rio Grande do Sul, Brasil, um estudo recente mostrou que $64,6 \%$ das mulheres de 20 a 49 anos, utilizam algum método anticoncepcional. O padrão de utilização de métodos nesta pesquisa foi diferente do Brasil como um todo, com $55,4 \%$ de usuárias de anticoncepcional oral, $22,2 \%$ de esterilizadas, $10,5 \%$ de usuárias de preservativo masculino e $7,7 \%$, DIU. O uso combinado de anticoncepcional oral e preservativo masculino contempla $1,0 \%$ da amostra. Outro dado importante revela que $22,2 \%$ das usuárias de anticoncepcional oral apresentavam alguma contra-indicação para seu consumo, das quais $10,8 \%$ fumavam e tinham idade igual ou maior que 35 anos, revelando falta de conhecimento sobre o uso adequado 5 .

Os estudos existentes sobre conhecimento avaliam populações específicas, como por exemplo: mulheres esterilizadas $6,7,8$, adolescentes $9,10,11$, mulheres em idade fértil $3,12,13$, mulheres com história de aborto 14 , além de dois estudos em homens universitários e de área rural 15,16. A maioria desses estudos identifica os métodos anticoncepcionais que a população conhece, mas não detalha o conhecimento sobre como utilizá-los e as contra-indicações de cada método $3,9,10,11,12,15$. Nesse contexto, a prevalência de conhecimento de algum método anticoncepcional encontrada no Brasil é elevada, acima de 80,0\% 3,9,15. Em Campinas, São Paulo, Espejo et al. 6 verificaram uma defasagem entre a referência espontânea aos distintos métodos contraceptivos e a qualidade deste conhecimento. Moraes Filho et al. 14 avaliaram que as mulheres pouco sabem a respeito dos métodos aos quais se referem espontaneamente. De modo semelhante, Vieira 8 encontrou um baixo índice de conhecimento ao combinar os métodos conhecidos com os atributos desses métodos.

Hardy et al. 13 estudando a adequação de uso do anticoncepcional oral, constataram que mais de 40,0\% das usuárias possuíam um ou mais fatores de risco para o seu uso, mesmo quando a indicação e obtenção do método ocorriam em serviço de saúde.

Para Alouini et al. 17, um dos principais problemas relacionados ao uso do anticoncepcional oral é a falta de conhecimento sobre o procedimento correto ao esquecer de tomar um comprimido. De acordo com a Pesquisa Nacional de Demografia em Saúde, realizada em 1996, cerca de metade das mulheres que foram mães entre 1990 e 1995, teve uma gravidez indesejada (Ministério da Saúde. Mulher e saúde: planejamento familiar. http://portal.saude.gov. $\mathrm{br} / \mathrm{saude} /$ visao.cfm?id_area $=152$, acessado em 12/Ago/2003). Em um estudo no Município de Pelotas, em 1995, gravidez indesejada foi observada em $41,0 \%$ do total de mulheres entre 15 e 19 anos que estiveram grávidas 12. Forrest 18 concluiu que a elevada porcentagem de gravidez indesejada, além de estar relacionada ao não-uso de contraceptivos, também pode ocorrer pelo uso incorreto destes. Nos resultados desta pesquisa, $47,0 \%$ dos casos de gravidez indesejada aconteceram entre as mulheres que usavam algum método contraceptivo.

Ainda que alguns autores venham enfatizando a importância da participação masculina no planejamento familiar e na anticoncepção 15,16,19,20, de maneira geral a responsabilidade ainda recai quase exclusivamente sobre a mulher 2. A falta de estudos sobre uso correto e adequado de métodos anticoncepcionais por parte dos homens dificulta o estabelecimento de estratégias para modificar esta realidade. Pascotto \& Sant'Ana 11, questionando estudantes adolescentes em relação à indicação de uso do preservativo masculino, encontraram que $62,1 \%$ desconheciam sua utilização como método de prevenção às doenças sexualmente transmissíveis. Por outro lado, como afirmam Duarte et al. 20, a descontinuidade do uso de métodos anticoncepcionais de participação masculina é elevada. Cerca de $60,0 \%$ dos usuários de abstinência periódica e de preservativo masculino, descontinuam o uso já no primeiro ano. A principal razão, conforme os autores, é a alta taxa de falha desses métodos.

Alguns estudos 10,11,21 revelam a falta de conhecimento sobre o ciclo menstrual e período fértil da mulher, fato que pode estar intimamente relacionado com a falha no uso do anticoncepcional oral e da abstinência periódica. Neste contexto, as mulheres optam pelo método irreversível. Um agravante para esta situação é que estudos realizados no Estado de São Paulo têm indicado uma proporção de arrependimento das mulheres que foram esterilizadas variando entre 10,0 e $20,0 \%$. A realização da operação antes dos 25 anos de idade, infor- 
mação deficiente acerca da esterilização feminina e o conhecimento de um menor número de métodos anticoncepcionais são os principais fatores de risco para o arrependimento 8,22.

Em razão disso, este estudo buscou avaliar o conhecimento sobre uso correto, indicações, contra-indicações e reversibilidade dos métodos anticoncepcionais mais utilizados pela população (anticoncepcional oral, esterilização feminina e preservativo masculino), bem como, aspectos fisiológicos do ciclo menstrual.

\section{Metodologia}

Realizou-se um estudo transversal de base populacional com homens e mulheres de 15 anos ou mais, residentes na zona urbana da Cidade de Pelotas. A pesquisa foi desenvolvida em conjunto por 16 mestrandos do Programa de Pós-graduação em Epidemiologia, Faculdade de Medicina, Universidade Federal de Pelotas, constituída de um instrumento único que foi aplicado à população, contendo questões gerais e específicas de cada pesquisador.

Em função das necessidades da pesquisa, estimou-se uma amostra de aproximadamente 1.440 domicílios com 3.456 pessoas na faixa etária em estudo. O processo de amostragem foi em múltiplos estágios. Os conglomerados foram definidos por meio da grade de setores censitários do Censo Demográfico 2000, do Instituto Brasileiro de Geografia e Estatística - IBGE (http://www.ibge.gov.br, acessado em 03/Set/ 2003). Os 409 setores censitários da zona urbana do município foram estratificados de acordo com a renda média do responsável pelo domicílio, e 144 setores foram sorteados sistematicamente. De posse da listagem dos domicílios elegíveis em cada setor sorteado, foram selecionados sistematicamente os domicílios proporcionalmente ao tamanho de cada setor, chegando-se a um total de 1.530 domicílios. Nestes, foram selecionados todos os indivíduos elegíveis na faixa etária de interesse.

O trabalho de campo foi desenvolvido entre outubro e dezembro de 2003 tendo a participação de 32 entrevistadoras com ensino médio completo. A seleção das entrevistadoras foi feita mediante treinamento específico de quarenta horas, aplicação de prova teórica e realização de entrevista domiciliar supervisionada, realizada em um setor censitário não incluído na amostra.

Realizou-se entrevista domiciliar utilizando um questionário estruturado e pré-codificado. Solicitou-se aos entrevistados um consentimento verbal informado. No caso de sujeitos au- sentes no momento da visita, as entrevistadoras retornaram pelo menos duas vezes a cada domicílio. Em caso de recusa, a última visita era realizada pelo supervisor de campo.

As variáveis demográficas avaliadas foram: sexo; idade (em anos completos); cor da pele (observada pela entrevistadora e classificada como branca, parda ou negra) e situação conjugal atual (com ou sem companheiro na época do estudo). Foram medidas as variáveis sócio-econômicas: escolaridade (em anos completos de estudo); renda familiar (renda dos moradores do domicílio no último mês medida de forma contínua e depois categorizada em número de salários mínimos nacionais na época do estudo - R\$240,00); nível econômico definido pela Associação Nacional de Empresas de Pesquisa - ANEP 23 (dividido em cinco categorias, de A a E, conforme escore) e trabalho fora de casa (sim ou não). Como variável comportamental foi avaliada a prática de religião (categorizada em católica, evangélica, espírita, outra e não praticante). Como variáveis reprodutivas, investigaram-se o número de filhos, idade quando o entrevistado teve o primeiro filho e gravidez indesejada. Avaliou-se, também, nos casos em que a gravidez indesejada ocorreu, se esta aconteceu na vigência de uso de algum método. Foi também medida a utilização de algum método anticoncepcional alguma vez na vida (categorizada posteriormente em números de métodos utilizados e combinações dos métodos mais utilizados) e a utilização de serviços de saúde como fonte de obtenção de informações na opção do último método utilizado (serviço de saúde público ou privado).

Para definir o desfecho, foi elaborado um questionário composto por dez questões de escolha simples, que contemplam o conhecimento sobre uso correto e adequado dos métodos anticoncepcionais mais utilizados pela população e aspectos fisiológicos do ciclo menstrual. Um escore de conhecimento foi obtido pelo número de respostas corretas, com peso igual para cada pergunta, podendo a pontuação total do escore variar de 0 (zero) a 10 (dez). A seleção das perguntas que definiram o escore foi feita com base nos métodos anticoncepcionais mais utilizados pela população de Pelotas 5 . Deu-se preferência às perguntas já testadas em outros estudos 7,8,11,12, enquanto as perguntas novas foram testadas e aperfeiçoadas em estudos piloto.

Os questionários foram revisados e codificados pelas entrevistadoras logo após as entrevistas e posteriormente pelo supervisor. Este realizou também a revisita em $10,0 \%$ da amostra, selecionada aleatoriamente, a qual consis- 
tia na aplicação de um questionário resumido para controle de qualidade e análise de concordância para obtenção do índice kappa. Este índice foi obtido por inetrmédio da variável gravidez indesejada. Entre os homens o kappa foi medido por meio da pergunta: “o Sr. já engravidou alguém que não queria ou não podia estar grávida?”. Entre as mulheres, a pergunta utilizada foi: "a Sra. já esteve grávida alguma vez que não queria ou não podia estar grávi$d a$ ?". Para ambas as questões as alternativas de resposta eram: sim, não e não soube informar. Não foi avaliado o índice kappa de questões do escore de conhecimento porque este pode modificar mesmo em curto espaço de tempo. Os dados foram digitados utilizando-se o programa Epi Info 6.04, com checagem automática de consistência e dupla digitação, para correção de possíveis erros de digitação. A análise dos dados foi realizada no programa estatístico Stata 8.0

A análise descritiva caracterizou a população geral e estratificada por sexo, conforme variáveis demográficas, sócio-econômicas, de comportamento, reprodutivas, de utilização de métodos anticoncepcionais e de caracterização da fonte de obtenção de informações (serviços de saúde públicos ou privados). O conjunto de perguntas que deram origem ao escore de conhecimento foi também analisado e a prevalência de acerto para cada questão foi descrita.

Posteriormente, levando em conta a distribuição normal do desfecho, realizou-se a análise pela regressão linear bivariada e análise de variância, testando-se a associação entre as exposições em estudo e o escore de conhecimento. $\mathrm{Na}$ análise bivariada foi verificada a tendência linear das médias para as variáveis ordinais e realizado o teste de heterogeneidade de médias para as variáveis categóricas. Os determinantes do conhecimento sobre métodos anticoncepcionais são diferentes entre homens e mulheres, assim as análises foram estratificadas por sexo. A análise ajustada foi subsidiada por modelo conceitual.

O modelo proposto compreende três níveis de determinação. No nível mais distal encontram-se as variáveis demográficas (sexo, idade, cor da pele e situação conjugal) juntamente com as sócio-econômicas (escolaridade, nível econômico e trabalho fora de casa). No segundo nível estão as variáveis reprodutivas (número de filhos, idade quando teve o primeiro filho e gravidez indesejada) juntamente com práticas religiosas. Estas, por sua vez, podem determinar a utilização de algum contraceptivo alguma vez na vida ou conduzir ao conhecimento levando à utilização destes (variáveis do ter- ceiro nível). Nesta análise o efeito de cada preditor é controlado para outras variáveis do mesmo nível e aquelas que se encontram no nível superior 24. Foram considerados fatores de confusão as variáveis associadas à exposição e ao desfecho com $\mathrm{p} \leq 0,2$. O escore foi tratado de forma contínua (0-10) e o efeito de delineamento amostral (EDA) foi considerado utilizandose o conjunto de comandos $s v y$, específico para a análise de inquéritos baseados em amostras complexas do programa estatístico Stata 8.0.

Esta pesquisa foi submetida e aprovada pela Comissão de Ética e Pesquisa da Universidade Federal de Pelotas.

\section{Resultados}

Foram entrevistados 3.542 indivíduos de 15 anos ou mais em 1.530 domicílios. O estudo apresentou um percentual de perdas e recusas de $3,4 \%$. A amostra obtida permitiu um poder estatístico de $80,0 \%$ para detectar diferenças de médias iguais ou maiores que 0,20 (desvio padrão $-\mathrm{dp}=1,92$ ) para o sexo masculino e 0,19 $(\mathrm{dp}=2,10)$ para o sexo feminino, em um nível de confiança de $95,0 \%$.

A Tabela 1 descreve a população estudada quanto a características demográficas, sócioeconômicas e de comportamento. A média de idade da amostra foi de 40 anos $(\mathrm{dp}=17,4)$, sendo $56,2 \%$ do sexo feminino. Observou-se que $80,2 \%$ eram de cor branca e $56,1 \%$ viviam com companheiro. Quanto à escolaridade, $24,0 \%$ possuíam até 4 anos, enquanto $13,1 \%$ tinham 12 anos ou mais de educação formal (média de escolaridade $=7,7 ; \mathrm{dp}=4,2$ ).

Quanto à renda familiar, 56,5\% possuíam uma renda mensal de até quatro salários mínimos, e $42,4 \%$ da amostra pertenciam aos níveis econômicos D e E. Com relação ao trabalho, entre os homens $62,6 \%$ trabalhavam fora de casa, enquanto, para as mulheres, este percentual foi de $36,9 \%$. Na população estudada, 45,6\% não praticavam religião; $26,7 \%$ eram católicos (Tabela 1).

A Tabela 2 apresenta a descrição das variáveis reprodutivas, utilização de métodos anticoncepcionais e de serviços de saúde como fonte de informação. O número médio de filhos por pessoa foi de $1,8(\mathrm{dp}=2,0) ; 1 / 3$ da amostra não tinha filhos; $14,2 \%$ possuíam quatro ou mais. Entre aqueles que tinham filhos, verificou-se uma maior proporção $(23,5 \%)$ de pessoas que tiveram o primeiro filho entre os $20 \mathrm{e}$ 24 anos de idade $(\mathrm{p}<0,001)$. O percentual de gravidez indesejada na amostra foi de $11,8 \%$. Entre as mulheres este percentual foi maior 
Tabela 1

Características da população estudada conforme variáveis demográficas, sócio-econômicas e de comportamento. Pelotas, Rio Grande do Sul, Brasil, 2003.

\begin{tabular}{|c|c|c|c|c|c|c|}
\hline \multirow[t]{2}{*}{ Variável } & \multicolumn{2}{|c|}{ Geral } & \multicolumn{2}{|c|}{ Homens } & \multicolumn{2}{|c|}{ Mulheres } \\
\hline & $\mathrm{n}^{*}, * *$ & $\%$ & $\mathrm{n}^{*}$ & $\%$ & $n^{*}$ & $\%$ \\
\hline Total & 3.542 & 100,0 & 1.551 & 43,8 & 1.991 & 56,2 \\
\hline \multicolumn{7}{|l|}{ Idade (anos) } \\
\hline $15-19$ & 442 & 12,5 & 207 & 13,4 & 235 & 11,8 \\
\hline $20-29$ & 759 & 21,4 & 342 & 22,0 & 417 & 20,9 \\
\hline $30-39$ & 645 & 18,2 & 279 & 18,0 & 366 & 18,4 \\
\hline $40-49$ & 680 & 19,2 & 303 & 19,5 & 377 & 18,9 \\
\hline $50-59$ & 493 & 13,9 & 213 & 13,7 & 280 & 14,1 \\
\hline 60 ou + & 523 & 14,8 & 207 & 13,4 & 316 & 15,9 \\
\hline \multicolumn{7}{|l|}{ Cor da pele } \\
\hline Branca & 2.841 & 80,2 & 1.234 & 79,6 & 1.607 & 80,7 \\
\hline Parda/negra & 701 & 19,8 & 317 & 20,4 & 384 & 19,3 \\
\hline \multicolumn{7}{|c|}{ Situação conjugal atual } \\
\hline Com companheiro & 1.989 & 56,1 & 971 & 62,6 & 1.018 & 51,1 \\
\hline Sem companheiro & 1.553 & 43,9 & 580 & 37,4 & 973 & 48,9 \\
\hline \multicolumn{7}{|c|}{ Escolaridade (anos de estudo) } \\
\hline 0 & 227 & 6,4 & 88 & 5,7 & 139 & 7,0 \\
\hline $1-4$ & 621 & 17,6 & 278 & 17,9 & 343 & 17,2 \\
\hline $5-8$ & 1.222 & 34,5 & 572 & 36,9 & 650 & 32,7 \\
\hline $9-11$ & 1.005 & 28,4 & 429 & 27,7 & 576 & 28,9 \\
\hline 12 ou + & 465 & 13,1 & 182 & 11,8 & 283 & 14,2 \\
\hline \multicolumn{7}{|c|}{ Renda familiar ${ }^{\star \star \star}$ (salários mínimos) } \\
\hline Até 1,0 & 452 & 12,9 & 181 & 11,8 & 271 & 13,7 \\
\hline $1,1-2,0$ & 543 & 15,4 & 227 & 14,7 & 316 & 15,9 \\
\hline $2,1-4,0$ & 995 & 28,2 & 460 & 29,9 & 535 & 27,0 \\
\hline $4,1-8,0$ & 843 & 23,9 & 362 & 23,5 & 481 & 24,2 \\
\hline+ de 8,0 & 692 & 19,6 & 310 & 20,1 & 382 & 19,2 \\
\hline \multicolumn{7}{|c|}{ Nível econômico (ANEP) 23} \\
\hline A & 177 & 5,0 & 85 & 5,5 & 92 & 4,6 \\
\hline B & 707 & 20,1 & 311 & 20,2 & 396 & 20,0 \\
\hline C & 1.146 & 32,5 & 508 & 33,0 & 638 & 32,2 \\
\hline D & 1.260 & 35,8 & 526 & 34,1 & 734 & 37,0 \\
\hline E & 234 & 6,6 & 111 & 7,2 & 123 & 6,2 \\
\hline \multicolumn{7}{|l|}{ Trabalho fora de casa } \\
\hline Sim & 1.705 & 48,1 & 971 & 62,6 & 734 & 36,9 \\
\hline Não & 1.837 & 51,9 & 580 & 37,4 & 1.257 & 63,1 \\
\hline \multicolumn{7}{|l|}{ Prática de religião } \\
\hline Não & 1.613 & 45,6 & 862 & 55,6 & 751 & 37,8 \\
\hline Católica & 943 & 26,7 & 336 & 21,7 & 607 & 30,5 \\
\hline Evangélica & 518 & 14,6 & 193 & 12,5 & 325 & 16,4 \\
\hline Espírita & 250 & 7,1 & 74 & 4,8 & 176 & 8,9 \\
\hline Outra & 211 & 6,0 & 84 & 5,4 & 127 & 6,4 \\
\hline
\end{tabular}

* Número de pessoas;

** O número máximo de valores ignorados foi de 18 para nível econômico

(ANEP - Associação Nacional de Empresas de Pesquisa 23);

*** Salário mínimo nacional na época do estudo igual a $R \$ 240,00$ (aproximadamente US\$80,00). 
Características da população estudada conforme variáveis reprodutivas, utilização de métodos anticoncepcionais e de serviços de saúde como fonte de informação. Pelotas, Rio Grande do Sul, Brasil, 2003.

\begin{tabular}{|c|c|c|c|c|c|c|}
\hline \multirow[t]{2}{*}{ Variável } & \multicolumn{2}{|c|}{ Geral } & \multicolumn{2}{|c|}{ Homens } & \multicolumn{2}{|c|}{ Mulheres } \\
\hline & $\mathrm{n}^{*}, * \star$ & $\%$ & $\mathrm{n}^{\star}$ & $\%$ & $\mathrm{n}^{*}$ & $\%$ \\
\hline Total & 3.542 & 100,0 & 1.551 & 43,8 & 1.991 & 56,2 \\
\hline \multicolumn{7}{|l|}{ Filhos } \\
\hline 0 & 1.179 & 33,3 & 557 & 36,0 & 622 & 31,3 \\
\hline 1 & 671 & 19,0 & 284 & 18,4 & 387 & 19,4 \\
\hline 2 & 735 & 20,8 & 319 & 20,6 & 416 & 20,9 \\
\hline 3 & 451 & 12,7 & 188 & 12,1 & 263 & 13,2 \\
\hline 4 ou + & 502 & 14,2 & 200 & 12,9 & 302 & 15,2 \\
\hline \multicolumn{7}{|l|}{ Idade quando teve $\circ 1$ o filho (anos) } \\
\hline $11-19$ & 627 & 17,8 & 128 & 8,3 & 499 & 25,2 \\
\hline $20-24$ & 828 & 23,5 & 356 & 23,2 & 472 & 23,9 \\
\hline $25-29$ & 533 & 15,2 & 277 & 18,0 & 256 & 12,9 \\
\hline $30 \mathrm{ou}+$ & 350 & 10,0 & 220 & 14,3 & 130 & 6,6 \\
\hline Não teve filhos & 1.179 & 33,5 & 557 & 36,2 & 622 & 31,4 \\
\hline \multicolumn{7}{|l|}{ Gravidez indesejada } \\
\hline Sim, utilizando algum método & 131 & 3,7 & 16 & 1,0 & 115 & 5,8 \\
\hline Sim, sem utilizar algum método & 283 & 8,1 & 77 & 5,0 & 206 & 10,4 \\
\hline Não & 3.102 & 88,2 & 1.442 & 94,0 & 1.660 & 83,8 \\
\hline \multicolumn{7}{|l|}{ Número de métodos utilizados ${ }^{\star \star \star}$} \\
\hline 0 & 875 & 24,7 & 482 & 31,2 & 393 & 19,7 \\
\hline 1 & 1.772 & 50,1 & 878 & 56,8 & 894 & 45,0 \\
\hline 2 & 743 & 21,0 & 153 & 9,9 & 590 & 29,6 \\
\hline 3 ou + & 147 & 4,2 & 33 & 2,1 & 114 & 5,7 \\
\hline \multicolumn{7}{|l|}{ Métodos utilizados ${ }^{\star \star \star}$} \\
\hline Nunca utilizou & 875 & 24,7 & 482 & 31,2 & 393 & 19,7 \\
\hline Anticoncepcional oral & 858 & 24,3 & 98 & 6,3 & 760 & 38,2 \\
\hline Preservativo masculino & 825 & 23,3 & 760 & 49,2 & 65 & 3,3 \\
\hline Anticoncepcional oral e preservativo masculino & 10462 & 13,1 & 118 & 7,6 & 344 & 17,3 \\
\hline Anticoncepcional oral e esterilização feminina & 115 & 3,3 & 3 & 0,2 & 112 & 5,6 \\
\hline Anticoncepcional oral e DIU & 76 & 2,1 & 5 & 0,3 & 71 & 3,5 \\
\hline Outras combinações de métodos & 326 & 9,2 & 80 & 5,2 & 246 & 12,4 \\
\hline \multicolumn{7}{|l|}{ Utilização de serviços de saúde\#\# } \\
\hline Não & 1.309 & 49,7 & 841 & 79,9 & 468 & 29,6 \\
\hline Público & 727 & 27,6 & 125 & 11,9 & 602 & 38,0 \\
\hline Privado & 600 & 22,7 & 87 & 8,2 & 513 & 32,4 \\
\hline
\end{tabular}

* Número de pessoas;

** O número máximo de valores ignorados foi de 26 para utilização de serviços de saúde e gravidez indesejada; *** Métodos utilizados ao longo da vida;

\# Variável sobre ter recebido informação sobre anticoncepção por profissional de saúde do setor público

ou privado, quando optou pelo último método utilizado, entre quem utilizava anticoncepcional $(n=2.662)$. 
( $\mathrm{p}<0,001$ ), alcançando $16,2 \%$. Destas, mais de $1 / 3$ estava utilizando algum método anticoncepcional.

$\mathrm{Na}$ amostra estudada, 75,3\% utilizaram algum método anticoncepcional alguma vez na vida, enquanto $31,2 \%$ dos homens e $19,7 \%$ das mulheres relataram nunca ter utilizado. Notou-se que $1 / 4$ da amostra utilizou dois ou mais métodos contraceptivos ao longo da vida. Entre as mulheres o método mais utilizado foi o anticoncepcional oral $(38,2 \%$ de uso exclusivo), enquanto entre os homens foi o preservativo masculino ( $49,2 \%$ de uso exclusivo). O uso destes dois métodos ao longo da vida é descrito por $13,1 \%$ da amostra, ficando cerca de 15,0\% distribuídos em outras combinações de métodos utilizados alguma vez na vida (Tabela 2).

Entre os usuários de métodos anticoncepcionais, $49,7 \%$ das pessoas que utilizam ou utilizaram alguma vez na vida, referiram não ter recebido informação sobre anticoncepção por profissional de saúde do setor público ou privado, quando optaram pelo último método utilizado, enquanto a obtenção desta informação no setor público foi relatada por $27,6 \%$ da amostra (Tabela 2). Entre estes, observou-se que 55,8\% pertenciam aos níveis econômicos $\mathrm{D}$ e $\mathrm{E}(\mathrm{p}<0,001) ; 1 / 4$ tinha até quatro anos de escolaridade $(\mathrm{p}<0,001)$.

A Tabela 3 mostra a prevalência de conhecimento sobre uso correto e adequado dos métodos anticoncepcionais para a amostra estudada e estratificada por sexo. As mulheres apresentaram prevalência de conhecimento superior aos homens para todas as questões $(\mathrm{p}<0,05)$, com exceção da pergunta sobre uso correto do preservativo masculino que foi respondida corretamente por $80,1 \%$ dos homens e $62,7 \% \mathrm{mu}$ lheres $(\mathrm{p}<0,01)$.

Pode ser observado que a grande maioria das mulheres $(86,9 \%)$ sabe que o anticoncepcional oral deve ser tomado todos os dias; porém, 70,0\% delas não referem corretamente o que devem fazer depois de esquecer de tomar um comprimido. A inadequação de uso do anticoncepcional oral por mulheres fumantes acima de 35 anos e por mulheres hipertensas ou com problemas cardíacos foi reconhecida, respectivamente, por $17,6 \%$ e $35,9 \%$ da população (Tabela 3).

Observa-se que $30,0 \%$ da amostra estudada não possuíam informação sobre a correta utilização do preservativo masculino e pouco mais da metade $(53,6 \%)$ identifica o uso deste como único método para prevenir DST. Chama a atenção que, apesar de 74,3\% conhecerem a indicação de uso da esterilização feminina, me- tade da amostra desconhece a dificuldade da reversão da cirurgia (Tabela 3 ).

Ainda na Tabela 3 pode-se perceber o elevado desconhecimento da população com relação ao ciclo menstrual e, principalmente, sobre o período fértil. Entre as mulheres, 44,7\% responderam corretamente a questão sobre o ciclo menstrual, enquanto que sobre o período fértil apenas 29,4\% acertaram.

A média de escore de conhecimento sobre métodos anticoncepcionais mais utilizados pela população na amostra foi de $4,65(\mathrm{dp}=2,07)$, com um efeito de delineamento de 3,70 e correlação intraclasse de 0,111 . As mulheres apresentaram um valor médio de 5,02 $(\mathrm{dp}=2,10)$, enquanto entre os homens este valor foi de 4,18 $(\mathrm{dp}=1,92)(\mathrm{p}<0,001)$

A Tabela 4 apresenta a análise bruta e ajustada dos fatores associados ao escore de conhecimento sobre métodos anticoncepcionais para homens. Após ajuste para fatores de confusão, a idade esteve inversamente associada ao escore de conhecimento, ao passo que escolaridade apresentou uma associação direta. Quem relatou gravidez indesejada apresentou um escore de conhecimento maior $(\beta=0,47$; intervalo de confiança de 95,0\% - IC95\%: 0,11$0,84)$. O uso de preservativo masculino exclusivo ou combinado com anticoncepcional oral, bem como outras combinações de métodos, estiveram associadas a um aumento no escore de conhecimento, apresentando coeficiente $\beta$ igual a 0,55 (IC95\%: 0,33-0,77); 0,52 (IC95\%: 0,15-0,89) e 1,08 (IC95\%: 0,64-1,51), respectivamente.

Entre as mulheres (Tabela 5), a análise ajustada mostrou um aumento linear significativo do escore de conhecimento com a idade; entretanto, o escore aumenta até a categoria de 30 a $39 \operatorname{anos}(\beta=1,77$; IC95\%: $1,46-2,08)$ e depois cai nas faixas etárias mais elevadas. Entre aquelas que vivem com companheiro, o escore de conhecimento foi maior ( $\beta=0,19$; IC95\%: 0,02-0,37). Observou-se uma associação linear direta entre escolaridade e escore de conhecimento; mulheres com 12 anos ou mais de estudo têm um acréscimo de 1,66 na média de escore quando comparadas àquelas sem escolaridade. Situação semelhante foi verificada com o nível econômico, em que o escore de conhecimento aumenta em direção aos estratos mais altos. O escore de conhecimento foi menor entre as mulheres católicas ou evangélicas, quando comparadas àquelas que não praticam religião. $\mathrm{O}$ uso de algum método anticoncepcional esteve associado a um aumento no escore de conhecimento, sendo maior entre aquelas que utilizaram anticoncepcional oral em combinação com um ou mais métodos. 
Prevalência de conhecimento da população sobre uso correto e adequado dos métodos anticoncepcionais mais utilizados e aspectos fisiológicos do ciclo menstrual ( $n=3.542$ ). Pelotas, Rio Grande do Sul, Brasil, 2003.

\begin{tabular}{|c|c|c|c|}
\hline \multirow[t]{2}{*}{ Variável } & \multicolumn{3}{|c|}{ Prevalências (\%) } \\
\hline & $\begin{array}{c}\text { Geral } \\
(n=3.542)\end{array}$ & $\begin{array}{l}\text { Homens } \\
(n=1.551)\end{array}$ & $\begin{array}{l}\text { Mulheres } \\
(n=1.991)\end{array}$ \\
\hline \multicolumn{4}{|c|}{$\begin{array}{l}\text { Se esquecer de tomar a pílula anticoncepcional um dia deve-se } \\
\text { tomar dois comprimidos juntos no dia seguinte no mesmo horário }\end{array}$} \\
\hline (1) $\operatorname{Sim}$ & 24,7 & 18,5 & 29,6 \\
\hline \multicolumn{4}{|c|}{$\begin{array}{l}\text { A pílula anticoncepcional deve ser tomada somente no dia ou } \\
\text { na hora em que vai acontecer a relação sexual }\end{array}$} \\
\hline (0) Não & 79,6 & 70,3 & 86,9 \\
\hline \multicolumn{4}{|c|}{ Mulheres que fumam e têm mais de 35 anos podem usar a pílula } \\
\hline (0) Não & 17,6 & 11,4 & 22,4 \\
\hline \multicolumn{4}{|c|}{$\begin{array}{l}\text { Mulheres que têm pressão alta ou problemas no coração } \\
\text { podem usar a pílula }\end{array}$} \\
\hline (0) Não & 35,9 & 31,0 & 39,6 \\
\hline \multicolumn{4}{|c|}{$\begin{array}{l}\text { Ao colocar a camisinha masculina deve-se apertar a ponta } \\
\text { para evitar que ela arrebente }\end{array}$} \\
\hline (1) $\operatorname{Sim}$ & 70,4 & 80,1 & 62,7 \\
\hline \multicolumn{4}{|c|}{$\begin{array}{l}\text { Além da camisinha masculina e feminina, existem outros métodos } \\
\text { anticoncepcionais que ajudam a prevenir tanto a gravidez quanto } \\
\text { doenças sexualmente transmissíveis (DST) }\end{array}$} \\
\hline (0) Não & 53,6 & 51,7 & 55,2 \\
\hline \multicolumn{4}{|c|}{$\begin{array}{l}\text { A ligadura de trompas é indicada exclusivamente para pessoas } \\
\text { que não querem ou não podem ter mais filhos }\end{array}$} \\
\hline (1) $\mathrm{Sim}$ & 74,3 & 69,7 & 77,8 \\
\hline \multicolumn{4}{|c|}{$\begin{array}{l}\text { Mulheres que tentam desfazer a ligadura de trompas raramente } \\
\text { conseguem ter mais filhos }\end{array}$} \\
\hline (1) $\operatorname{Sim}$ & 49,9 & 45,5 & 53,4 \\
\hline \multicolumn{4}{|l|}{ Quando começa um ciclo menstrual? } \\
\hline (1) No primeiro dia da menstruação & 35,6 & 23,9 & 44,7 \\
\hline \multicolumn{4}{|c|}{$\begin{array}{l}\text { Numa mulher cujo ciclo menstrual é de } 28 \text { dias, a maior } \\
\text { possibilidade de engravidar ocorre }\end{array}$} \\
\hline (3) No 14o dia após o início da menstruação & 23,4 & 15,7 & 29,4 \\
\hline
\end{tabular}

Nota: O teste do qui-quadrado para heterogeneidade de proporções entre os sexos apresentou valor $p<0,001$ para todas as questões, com exceção da questão sobre métodos anticoncepcionais e DST que apresentou $p=0,042$.

O diagnóstico do modelo de regressão linear por sexo foi realizado por meio do gráfico normal de resíduos, não apresentando desvio da linearidade.

O teste kappa obtido pela variável gravidez indesejada indicou uma concordância excelente, apresentando um índice de 0,82 para homens, enquanto entre as mulheres o índice foi ainda maior, 0,88.

\section{Discussão}

O estudo indica que, apesar da elevada prevalência de utilização de algum método anticoncepcional ao longo da vida $(75,3 \%)$, ainda é limitado o conhecimento sobre uso correto e adequado dos métodos mais utilizados, assim como sobre ciclo menstrual e período fértil. A média para o escore de conhecimento encontrada nesta população $(4,65)$ foi diferente entre homens $(4,18)$ e mulheres $(5,02)$. Os fatores as- 
Associação entre variáveis demográficas, sócio-econômicas, comportamentais, reprodutivas, utilização de métodos anticoncepcionais e escore de conhecimento para homens $(n=1.551)$. Pelotas, Rio Grande do Sul, Brasil, 2003.

\begin{tabular}{|c|c|c|c|c|c|c|}
\hline \multirow[t]{2}{*}{ Variável } & \multicolumn{3}{|c|}{ Análise bruta* } & \multicolumn{3}{|c|}{ Análise ajustada** } \\
\hline & Média & $\begin{array}{l}\text { Desvio } \\
\text { padrão }\end{array}$ & Valor $\mathrm{p}$ & B & IC95\%** & Valor $\mathrm{p}$ \\
\hline \multicolumn{7}{|l|}{ Nível 1} \\
\hline Total & 4,18 & 1,92 & & & & \\
\hline Idade (anos) & & & $<0,001^{\star \star \star}$ & & & $<0,001^{\star \star \star}$ \\
\hline $15-19$ & 4,21 & 1,74 & & 0,90 & $0,54-1,26$ & \\
\hline $20-29$ & 4,63 & 1,64 & & 1,14 & $0,80-1,47$ & \\
\hline $30-39$ & 4,51 & 1,72 & & 1,13 & $0,78-1,48$ & \\
\hline $40-49$ & 4,43 & 1,92 & & 1,12 & $0,78-1,46$ & \\
\hline $50-59$ & 3,90 & 1,92 & & 0,66 & $0,31-1,01$ & \\
\hline 60 ou + & 2,87 & 2,17 & & & & \\
\hline Escolaridade (anos de estudo) & & & $<0,001 * \star \star$ & & & $<0,001^{\star \star \star}$ \\
\hline 0 & 2,63 & 2,16 & & & & \\
\hline $1-4$ & 3,37 & 1,89 & & 0,37 & $0,06-0,80$ & \\
\hline $5-8$ & 3,98 & 1,76 & & 0,84 & $0,41-1,27$ & \\
\hline $9-11$ & 4,81 & 1,67 & & 1,54 & $1,07-2,00$ & \\
\hline $12 \mathrm{ou}+$ & 5,34 & 1,65 & & 1,95 & $1,42-2,48$ & \\
\hline Nível econômico (ANEP 23) & & & $<0,001^{\star \star \star}$ & & & $0,056^{\star \star \star}$ \\
\hline A & 5,33 & 1,74 & & 0,41 & $-0,16-0,98$ & \\
\hline B & 4,62 & 1,79 & & 0,13 & $-0,30-0,56$ & \\
\hline $\mathrm{C}$ & 4,28 & 1,89 & & 0,05 & $-0,34-0,47$ & \\
\hline $\mathrm{D}$ & 3,78 & 1,91 & & $-0,09$ & $-0,46-0,28$ & \\
\hline$E$ & 3,53 & 1,88 & & & & \\
\hline Trabalho fora de casa & & & $<0,001 \#$ & & & $0,174 \#$ \\
\hline Sim & 4,38 & 1,84 & & 0,14 & $-0,06-0,34$ & \\
\hline Não & 3,83 & 2,01 & & & & \\
\hline \multicolumn{7}{|l|}{ Nível 2} \\
\hline Prática de religião & & & $0,002 \#$ & & & $0,160 \#$ \\
\hline Não & 4,32 & 1,93 & & - & & \\
\hline Católica & 3,93 & 1,97 & & $-0,26$ & $-0,48--0,03$ & \\
\hline Evangélica & 3,91 & 1,89 & & $-0,16$ & $-0,43-0,12$ & \\
\hline Espírita & 4,61 & 1,58 & & 0,06 & $-0,36-0,42$ & \\
\hline Outra & 3,98 & 1,79 & & $-0,25$ & $-0,64-0,14$ & \\
\hline Idade em que teve o 1ㅇ filho (anos) & & & $0,062^{\star \star \star}$ & & & $0,138^{\star \star \star}$ \\
\hline $11-19$ & 3,95 & 1,71 & & & & \\
\hline $20-24$ & 4,22 & 1,94 & & 0,49 & $0,12-0,85$ & \\
\hline $25-29$ & 4,04 & 2,05 & & 0,33 & $-0,05-0,71$ & \\
\hline $30 \mathrm{ou}+$ & 4,10 & 2,00 & & 0,45 & $0,05-0,86$ & \\
\hline Não teve filhos & 4,33 & 1,83 & & $-0,02$ & $-0,39-0,35$ & \\
\hline Gravidez indesejada & & & $<0,001 \#$ & & & $0,011 \#$ \\
\hline Sim & 4,72 & 1,44 & & 0,47 & $0,11-0,84$ & \\
\hline Não & 4,15 & 1,94 & & & & \\
\hline \multicolumn{7}{|l|}{ Nível 3} \\
\hline Métodos utilizados & & & $<0,001 \#$ & & & $<0,001 \#$ \\
\hline Nunca utilizou & 3,37 & 2,10 & & & & \\
\hline Anticoncepcional oral & 4,08 & 1,60 & & 0,31 & $-0,07-0,69$ & \\
\hline Preservativo masculino & 4,51 & 1,73 & & 0,55 & $0,33-0,77$ & \\
\hline $\begin{array}{l}\text { Anticoncepcional oral } \\
\text { e preservativo masculino }\end{array}$ & 4,67 & 1,62 & & 0,52 & $0,15-0,89$ & \\
\hline $\begin{array}{l}\text { Anticoncepcional oral } \\
\text { e esterilização feminina }\end{array}$ & 4,00 & 1,00 & & $-0,40$ & $-2,36-1,55$ & \\
\hline Anticoncepcional oral e DIU & 5,00 & 1,41 & & 0,77 & $-0,75-2,29$ & \\
\hline Outras combinações de métodos & 5,25 & 1,69 & & 1,08 & $0,64-1,51$ & \\
\hline
\end{tabular}

$B=$ coeficiente $B ;$ IC95\%: intervalo de confiança de $95,0 \%$;

* Cor da pele, situação conjugal atual e número de filhos não se mantiveram no modelo final $(p>0,2)$;

** Cada variável está ajustada para as demais do mesmo nível e para as dos níveis acima

(foram mantidas no modelo as variáveis com valor $p \leq 0,2$ );

*** Teste de Wald para tendência linear;

\# Teste de Wald para heterogeneidade de médias. 
Associação entre variáveis demográficas, sócio-econômicas, comportamentais, reprodutivas, utilização de métodos anticoncepcionais e escore de conhecimento para mulheres $(n=1.991)$. Pelotas, Rio Grande do Sul, Brasil, 2003.

\begin{tabular}{|c|c|c|c|c|c|c|}
\hline \multirow[t]{2}{*}{ Variável } & \multicolumn{3}{|c|}{ Análise bruta* } & \multicolumn{3}{|c|}{ Análise ajustada ${ }^{\star \star}$} \\
\hline & Média & $\begin{array}{l}\text { Desvio } \\
\text { padrão }\end{array}$ & Valor $p$ & B & IC95\%** & Valor $\mathrm{p}$ \\
\hline \multicolumn{7}{|l|}{ Nível 1} \\
\hline Total & 5,02 & 2,10 & & & & \\
\hline Idade (anos) & & & $<0,001 * \star \star$ & & & $<0,001^{\star \star *}$ \\
\hline $15-19$ & 5,03 & 1,73 & & 1,42 & $1,08-1,75$ & \\
\hline $20-29$ & 5,59 & 1,72 & & 1,72 & $1,41-2,02$ & \\
\hline $30-39$ & 5,56 & 1,88 & & 1,77 & $1,46-2,08$ & \\
\hline $40-49$ & 5,53 & 1,83 & & 1,71 & $1,40-2,01$ & \\
\hline $50-59$ & 4,93 & 2,18 & & 1,30 & $0,99-1,61$ & \\
\hline $60 \mathrm{ou}+$ & 3,11 & 2,18 & & & & \\
\hline Cor da pele & & & $<0,001 \#$ & & & $0,050 \#$ \\
\hline Branca & 5,12 & 2,15 & & 0,21 & $0,00-0,42$ & \\
\hline Parda/negra & 4,60 & 1,86 & & & & \\
\hline Situação conjugal atual & & & $<0,001 \#$ & & & $0,032 \#$ \\
\hline Com companheiro & 5,24 & 1,96 & & 0,19 & $0,02-0,37$ & \\
\hline Sem companheiro & 4,79 & 2,22 & & & & \\
\hline Escolaridade (anos de estudo) & & & $<0,001^{\star * \star}$ & & & $<0,001^{\star \star \star}$ \\
\hline 0 & 2,92 & 2,13 & & & & \\
\hline $1-4$ & 4,00 & 2,09 & & 0,40 & $0,03-0,77$ & \\
\hline $5-8$ & 4,90 & 1,85 & & 0,94 & $0,57-1,30$ & \\
\hline $9-11$ & 5,71 & 1,74 & & 1,43 & $1,03-1,83$ & \\
\hline 12 ou + & 6,16 & 1,96 & & 1,66 & $1,20-2,11$ & \\
\hline Nível econômico (ANEP 23) & & & $<0,001$ *** & & & $<0,001^{\star \star \star}$ \\
\hline A & 6,01 & 2,02 & & 0,59 & $0,04-1,13$ & \\
\hline B & 5,74 & 2,00 & & 0,60 & $0,19-1,02$ & \\
\hline C & 5,31 & 1,95 & & 0,44 & $0,07-0,82$ & \\
\hline D & 4,43 & 2,05 & & 0,03 & $-0,32-0,38$ & \\
\hline$E$ & 4,00 & 2,12 & & & & \\
\hline Trabalho fora de casa & & & $<0,001 \#$ & & & $0,146 \#$ \\
\hline Sim & 5,58 & 1,87 & & 0,14 & $-0,04-0,33$ & \\
\hline Não & 4,69 & 2,16 & & & & \\
\hline \multicolumn{7}{|l|}{ Nível 2} \\
\hline Prática de religião & & & $<0,001 \#$ & & & $<0,001 \#$ \\
\hline Não & 5,34 & 1,97 & & & & \\
\hline Católica & 4,77 & 2,18 & & $-0,31$ & $-0,51--0,11$ & \\
\hline Evangélica & 4,46 & 2,11 & & $-0,38$ & $-0,63--0,14$ & \\
\hline Espírita & 5,80 & 1,89 & & 0,21 & $-0,09-0,52$ & \\
\hline Outra & 4,65 & 2,14 & & $-0,29$ & $-0,64-0,06$ & \\
\hline Gravidez indesejada & & & $0,874 \#$ & & & $0,074 \#$ \\
\hline Sim & 5,04 & 2,01 & & 0,20 & $-0,02-0,42$ & \\
\hline Não & 5,02 & 2,12 & & & & \\
\hline \multicolumn{7}{|l|}{ Nível 3} \\
\hline Métodos utilizados & & & $<0,001 \#$ & & & $<0,001 \#$ \\
\hline Nunca utilizou & 3,59 & 2,31 & & & & \\
\hline Anticoncepcional oral & 5,06 & 1,87 & & 0,85 & $0,60-1,10$ & \\
\hline Preservativo masculino & 5,12 & 2,00 & & 0,95 & $0,47-1,42$ & \\
\hline $\begin{array}{l}\text { Anticoncepcional oral } \\
\text { e preservativo masculino }\end{array}$ & 5,85 & 1,58 & & 1,24 & $0,95-1,52$ & \\
\hline $\begin{array}{l}\text { Anticoncepcional oral } \\
\text { e esterilização feminina }\end{array}$ & 5,51 & 1,90 & & 1,11 & $0,70-1,52$ & \\
\hline Anticoncepcional oral e DIU & 5,56 & 2,07 & & 1,11 & $0,63-1,58$ & \\
\hline Outras combinações de métodos & 5,61 & 2,08 & & 1,14 & $0,83-1,46$ & \\
\hline
\end{tabular}

$B=$ coeficiente $B ;$ IC95\%: intervalo de confiança de $95,0 \%$;

* Idade quando teve o 1 o filho e número de filhos não se mantiveram no modelo final $(p>0,2)$;

** Cada variável está ajustada para as demais do mesmo nível e para as dos níveis acima

(foram mantidas no modelo as variáveis com valor $p \leq 0,2$ );

*** Teste de Wald para tendência linear;

\# Teste de Wald para heterogeneidade de médias. 
sociados ao conhecimento também foram distintos conforme o sexo. Para os homens, a menor idade, maior escolaridade, relato de gravidez indesejada e uso de método anticoncepcional exclusivo ou combinado ao longo da vida mostraram-se associados a um maior escore de conhecimento. Entre as mulheres, os determinantes do maior escore de conhecimento foram maior idade, viver com companheiro, maior escolaridade, melhor nível econômico, não ter religião e uso de método anticoncepcional exclusivo ou combinado ao longo da vida.

Avaliando a validade dos achados, percebese que o estudo utilizou delineamento transversal, que proporciona rapidez e baixo custo na realização da pesquisa; contudo, uma vez que as informações sobre exposição e desfecho são coletadas no mesmo momento, pode ser afetado por causalidade reversa. Este viés pode ter afetado a associação de algumas exposições, sobretudo variáveis reprodutivas e de utilização de métodos anticoncepcionais, com o escore de conhecimento.

O baixo percentual de perdas e recusas $(3,4 \%)$ e o perfil sócio-demográfico desta amostra, condizentes com o do censo populacional de Pelotas (Censo Demográfico 2000), suportam a representatividade da amostra para a população de 15 anos ou mais residente na zona urbana da cidade.

Um dado que chama a atenção no perfil de utilização de serviços de saúde como fonte de obtenção de informações sobre anticoncepção é que metade dos usuários de métodos anticoncepcionais referiu não ter obtido informações sobre anticoncepção em serviço de saúde público ou privado quando escolheu o último método utilizado, indicando que a escolha não foi orientada por profissional de saúde. Observa-se, também, que pouco mais de $1 / 4$ da amostra relatou ter recebido informação sobre contracepção no setor público, o que indica que este setor desempenha um papel pequeno na orientação da escolha do método e, conseqüentemente, da sua forma de utilização. Para o Estado de São Paulo, Hardy et al. 13 descreveram que $35,0 \%$ das mulheres da amostra iniciaram o uso do anticoncepcional oral sem consulta prévia, enquanto 1/4 disse ter consultado previamente um serviço público de saúde. Um estudo realizado em Pelotas encontrou que a escolha do tipo de serviço de saúde depende mais da classe social e escolaridade que da gravidade dos problemas de saúde 25. Verificando-se que mais da metade da população que utiliza o setor público de saúde como fonte de informação sobre métodos anticoncepcionais pertence ao nível econômico D e E, e 1/4 tem até quatro anos de escolaridade, percebe-se a lei dos cui- dados inversos em saúde, em que aqueles indivíduos com maior necessidade, ou seja, que dependem do SUS, são os que têm menos acesso à informação adequada.

Conforme a literatura revisada, não existem estudos de base populacional que abordem o conhecimento sobre métodos anticoncepcionais em indivíduos de ambos os sexos, assim como os fatores associados a este conhecimento. A maioria dos estudos avalia o conhecimento acerca das distintas opções de métodos anticoncepcionais existentes, sem avaliar o conhecimento sobre o uso correto e adequado de cada método $3,9,10,11,12,15$. Além disso, os estudos em geral dizem respeito a populações específicas 3,6,7,8,9,10,11,12,13,14,15,16.

Um dos poucos estudos encontrados que avaliou a qualidade da informação acerca dos métodos citados utilizou um escore para caracterizar o conhecimento de uma amostra de mulheres esterilizadas e não esterilizadas. O conhecimento foi inadequado para $52,4 \%$ da amostra 6 . Outro estudo em mulheres esterilizadas com menos de quarenta anos combinou o número de métodos conhecidos com o conhecimento de alguns atributos desses métodos e construiu um índice para medir o conhecimento, classificando-o como baixo para $24,0 \%$, médio para $40,0 \%$ e elevado para $36,0 \%$ da amostra 8 . Moraes Filho et al. 14 verificaram um menor escore de conhecimento sobre métodos anticoncepcionais referidos espontaneamente por mulheres com aborto provocado comparadas àquelas que tiveram aborto espontâneo.

A diferença entre a amostra de base populacional estudada e as populações avaliadas nos outros estudos sobre este tema, assim como os distintos critérios utilizados na avaliação do conhecimento, limitam a comparabilidade dos resultados.

Avaliando as perguntas que formaram o escore de conhecimento, percebe-se a escassa informação da população sobre este tema. $\mathrm{Na}$ França 17, em um estudo com baixa taxa de resposta, $30,0 \%$ das mulheres desconheciam o procedimento após esquecer de tomar um comprimido do anticoncepcional oral. Neste estudo o desconhecimento foi ainda maior. O conhecimento sobre a adequação de uso deste método foi consistente com estudos realizados em Pelotas 5 e São Paulo 13, que indicaram, respectivamente, $22,2 \%$ e $40,0 \%$ de uso inadequado para as usuárias de anticoncepcional oral, mostrando a escassa percepção dessas mulheres sobre a indicação de uso deste método.

Não obstante as campanhas educativas em relação ao uso do preservativo masculino, ainda é insuficiente o conhecimento sobre sua cor- 
reta utilização para maior efetividade. Estudo realizado com estudantes adolescentes no $\mathrm{Pa}$ raná 11 aponta que $37,0 \%$ reconhecem a vantagem profilática do preservativo masculino, sendo esta prevalência menor do que a encontrada nesta pesquisa.

Em um estudo de conhecimento sobre métodos anticoncepcionais em mulheres esterilizadas, cerca de um terço discordou ou não sabia da dificuldade da reversão da esterilização feminina 7 . A dificuldade de reversão dessa cirurgia, no presente estudo, não foi percebida por metade da amostra, situação que sugere a grande falta de informação sobre este método e pode levar ao arrependimento em mulheres que se esterilizam sem este conhecimento prévio.

No tocante a aspectos fisiológicos do ciclo menstrual, mais da metade das mulheres desconhece o início do ciclo; este resultado é consistente com Pascotto \& Sant'Ana 11 e pode provocar o uso incorreto do anticoncepcional oral. O desconhecimento sobre período fértil do ciclo menstrual nesta pesquisa concorda com outros estudos sobre este tema 10,11,26,27 e compromete o uso correto do método de abstinência periódica.

Analisando o escore de conhecimento, observa-se que as mulheres apresentaram uma média de escore superior aos homens. Este achado pode ser atribuído ao fato de que a maioria dos métodos anticoncepcionais depende apenas da participação feminina, sendo comum o entendimento de que só a mulher é responsável pela anticoncepção 2 , ficando a participação masculina restrita normalmente a impossibilidade de a mulher de usar algum método contraceptivo 20 . Um estudo multicêntrico, realizado em quatro países da África e um da América Central, mostrou, na maioria dos países da amostra, nível de conhecimento similar sobre a indicação do uso do preservativo masculino e período fértil do ciclo menstrual 27. Este resultado diferente pode ser justificado por especificidades culturais daqueles países.

$\mathrm{O}$ aumento da idade esteve associado à diminuição do escore de conhecimento entre os homens. Entre as mulheres, houve um aumento linear significativo com a idade; entretanto, a partir dos quarenta anos apresentam um leve decréscimo em direção às faixas etárias mais elevadas. Resultado semelhante foi encontrado em estudo que detectou um escore de conhecimento maior para as mulheres de 30 a 39 anos quando comparadas às da faixa etária de 40 a 49 anos, embora este resultado não tenha sido estatisticamente significativo 6 . O declínio do escore de conhecimento, conforme aumenta a idade nos homens, e o baixo escore de conhe- cimento das mulheres acima de quarenta anos pode estar relacionado às mudanças importantes na saúde reprodutiva nas duas últimas décadas 1 . Este efeito pode ser explicado pelo aumento da utilização de métodos anticoncepcionais incluindo os de participação masculina, especialmente vasectomia e preservativo masculino 1,20. A epidemia da AIDS provocou a disseminação de discussões sobre sexualidade, prevenção de DST e contracepção, tendo um impacto marcante na utilização de anticoncepcionais. Tais aspectos estão intimamente relacionados ao aumento do conhecimento 28 .

Entre as mulheres que vivem com companheiro o escore de conhecimento foi maior quando comparadas àquelas sem companheiro, contrariando o resultado de outro estudo em que não ocorreu associação entre situação conjugal e conhecimento 6 . A associação encontrada neste estudo é plausível, uma vez que mulheres que vivem com companheiro são, em geral, mais expostas à atividade sexual e, por esta razão, expostas também à prática da anticoncepção.

A escolaridade esteve diretamente associada ao escore de conhecimento em homens e mulheres, ou seja, quanto maior a escolaridade do indivíduo, mais elevado é o escore. O efeito da escolaridade sobre o escore de conhecimento encontrado neste estudo é sustentado por várias pesquisas, mesmo aquelas que apenas avaliaram o conhecimento geral sobre algum método 6,13,21. Um destes estudos 21 foi realizado nos Estados Unidos em 2002 e observou que quanto menor a escolaridade, maior o uso incorreto do anticoncepcional oral e do preservativo masculino, levando à falha destes métodos e, por conseguinte, aumentando a probabilidade de uma gravidez indesejada. Em São Paulo, Hardy et al. 13 observaram uma relação inversa entre a percentagem de mulheres com risco para o uso do anticoncepcional oral e a escolaridade da mulher.

O estudo mostrou que mulheres com nível econômico mais alto apresentam um maior escore de conhecimento, o que é consistente com o estudo de Espejo et al. 6. Este resultado evidencia a relação de fatores sócio-econômicos com o acesso à informação sobre anticoncepção, apontada também pela escolaridade.

Mulheres católicas ou evangélicas apresentaram um menor escore de conhecimento. Uma explicação para este achado é que as religiões não aprovam o uso de contraceptivos e a utilização de métodos possui uma relação estreita com o conhecimento 12 .

Gravidez indesejada demonstrou associação significativa com um maior escore de conhecimento somente para os homens. Não foi encontrado outro estudo investigando tal associação. 
Este resultado pode indicar que os homens procuram informação sobre anticoncepção após vivenciarem uma situação de gravidez não desejada, ou de outro modo, procuram tomar para si a responsabilidade de evitar uma nova gravidez. Todavia, a pergunta sobre gravidez indesejada, ainda que apresente boa reprodutibilidade indicada pelo kappa, aborda um assunto íntimo e pode ter sido afetada por um viés de informação do entrevistado. Este viés pode subestimar a prevalência, tanto para homens quanto para mulheres.

Alguns estudos 12,28 salientam a utilização prévia de algum método anticoncepcional associada a um maior conhecimento. Este resultado é consistente com o presente estudo, em que a utilização de algum método anticoncepcional na vida esteve associada a um maior escore de conhecimento entre homens e mulheres. Uma possível limitação nesta abordagem, é que o número de métodos anticoncepcionais utilizados tenha sido afetado por viés de memória, visto que os indivíduos com maior conhecimento sobre anticoncepção podem lembrar melhor quais os métodos utilizados ao longo da vida. Homens podem não ter referido o método de anticoncepção do casal quando este era feminino, e as mulheres podem não ter referido a laqueadura por não considerá-la um método anticoncepcional. O elevado escore de conhecimento observado em outras combinações de métodos está de acordo com a presen-

\section{Resumo}

A prevalência de uso de anticoncepcional no Brasil é alta; porém, é freqüente seu uso incorreto e inadequado, sugerindo escasso conhecimento sobre os métodos. Assim, avaliou-se o conhecimento sobre métodos anticoncepcionais mais utilizados por meio de um escore (0-10) e fatores associados a este conhecimento. Foi realizado um estudo transversal de base populacional, com 3.542 indivíduos de 15 anos ou mais, residentes na zona urbana de Pelotas, Rio Grande do Sul. A média de escore de conhecimento foi de 4,65 ( $d p=2,07)$, sendo $5,02(d p=2,10)$ para as mulheres e 4,18 $(d p=1,92) p a$ ra os homens. Menor idade, maior escolaridade, relato de gravidez indesejada e uso de método anticoncepcional exclusivo ou combinado ao longo da vida mostraram-se associados a um maior escore de conhecimento entre os homens, enquanto, para as mulheres, os determinantes de maior conhecimento foram maior idade, viver com companheiro, maior escolaridade, melhor nível econômico, não ter religião e uso de método anticoncepcional exclusivo ou combinado ao longo da vida. Apesar da alta prevalência de uso de anticoncepcional (75,3\%), é limitado o conhecimento sobre os métodos mais utilizados, ciclo menstrual e periodo fértil.

Conhecimentos, Atitudes e Prática em Saúde; Anticoncepção; Anticoncepcionais ça, nesta categoria, de métodos menos utilizados e que exigem um maior conhecimento para a sua utilização.

Os resultados deste estudo revelam limitado conhecimento acerca dos métodos mais utilizados e sobre período fértil do ciclo menstrual, apontando uma importante limitação do PAISM no que se refere à promoção de informações adequadas sobre planejamento familiar 2. A pesquisa também indica que o grupo populacional que necessita de ampliar seus conhecimentos sobre anticoncepção e, portanto, deve ser priorizado nas ações educativas a serem desenvolvidas pelo PAISM, é o segmento de baixo nível econômico e escolaridade. A escolaridade, por sua vez, deve ser considerada como um elemento importante no conhecimento sobre saúde reprodutiva, já que a baixa escolaridade pode levar a dificuldades na assimilação das informações recebidas, ao passo que a escolaridade maior melhora o acesso à informação, amplia a participação masculina na contracepção 19,20, criando assim melhores condições para o casal fazer a escolha contraceptiva adequada e a sua correta utilização. Por fim, o estudo demonstra que os homens também carecem de informação sobre anticoncepção, e, desse modo, a inclusão masculina na abordagem do planejamento familiar poderia ser uma nova estratégia a fim de melhorar o desempenho deste programa.

\section{Colaboradores}

V. M. V. Paniz participou da revisão de literatura, elaboração do projeto, elaboração do instrumento de coleta de dados, definição da amostra e logística, treinamento das entrevistadoras, supervisão da coleta de dados, análise dos dados, redação final do artigo. A. G. Fassa contribuiu na elaboração do projeto e do instrumento de coleta de dados, na análise dos dados e na redação do artigo final. M. C. Silva colaborou na elaboração do projeto e do instrumento de coleta de dados, análise dos dados, redação do artigo final.

\section{Agradecimentos}

Este trabalho contou com o apoio financeiro da Coordenação de Aperfeiçoamento de Pessoal de Nível Superior. 


\section{Referências}

1. Vieira EM, Badiani R, Dal Fabbro AL, Rodrigues Jr. AL. Características do uso de métodos anticoncepcionais no Estado de São Paulo. Rev Saúde Pública 2002; 36:263-70.

2. Osis M. Paism: um marco na abordagem da saúde reprodutiva no Brasil. Cad Saúde Pública 1998;14 Suppl 1:25-32.

3. Schor N, Ferreira AF, Machado VL, França AP, Pirotta KC, Alvarenga AT, et al. Mulher e anticoncepção: conhecimento e uso de métodos anticoncepcionais. Cad Saúde Pública 2000; 16:37784.

4. Monteiro CA. Velhos e novos males da saúde no Brasil: a evolução do país e de suas doenças. São Paulo: Editora Hucitec; 1995.

5. Dias-da-Costa JS, Gigante DP, Menezes AMB, Olinto MTA, Macedo S, Britto MAP, et al. Uso de métodos anticoncepcionais e adequação de contraceptivos hormonais orais na Cidade de Pelotas, Rio Grande do Sul, Brasil: 1992 e 1999. Cad Saúde Pública 2002; 18:93-9.

6. Espejo X, Tsunechiro MA, Osis MJD, Duarte GA, Bahamondese L, Sousa MH. Adequação do conhecimento sobre métodos anticoncepcionais entre mulheres de Campinas, São Paulo. Rev Saúde Pública 2003; 37:583-90.

7. Vieira EM. A esterilização de mulheres de baixa renda em região metropolitana do sudeste do Brasil e fatores ligados à sua prevalência. Rev Saúde Pública 1994; 28:440-8.

8. Vieira EM. O arrependimento após a esterilização feminina. Cad Saúde Pública 1998; 14 Suppl 1:5968.

9. Bruno ZV, Souza MA, Teixeira LGM, Silva RB, Silva RB, Guanabara EM, et al. Sexualidade e anticoncepção na adolescência: conhecimento e atitude. Reprod Clim 1997; 12:137-40.

10. Costa JO, Carvalho MAB, Garrido AQ, Gouvêa CML, Alves DS, Bolwerk GA, et al. Sexualidade na adolescência: conhecimentos, atitudes e práticas dos estudantes de Barbacena, 2001. Ginecol Obstet Atual 2003; 12:12-7.

11. Risso-Pascotto C, Sant'Ana DMG. Avaliação dos conhecimentos sobre métodos contraceptivos entre alunos do 1o e 3 o anos do ensino médio do Colégio Estadual de Umuarama - Ensino fundamental e médio - Umuarama (PR). Arq Ciências Saúde UNIPAR 1999; 3:143-51.

12. Olinto MTA, Galvão LW. Características reprodutivas de mulheres de 15 a 49 anos: estudos comparativos e planejamento de ações. Rev Saúde Pública 1999; 33:64-72.

13. Hardy EE, de Moraes TM, Faúndes A, Vera S, Pinotti JA. Adequação do uso de pílula anticoncepcional entre mulheres unidas. Rev Saúde Pública 1991; 25:96-102.
14. Moraes Filho OB, Albuquerque RM, Hardy EE. Conhecimento e uso de métodos anticoncepcionais por mulheres com aborto provocado ou espontâneo. Rev IMIP 1997; 11:32-40.

15. Duarte GA. Perspectiva masculina quanto a métodos contraceptivos. Cad Saúde Pública 1998; 14 Suppl 1:125-30.

16. Espirito-Santo DC, Tavares-Neto J. A visão masculina sobre métodos contraceptivos em uma comunidade rural da Bahia, Brasil. Cad Saúde Pública 2004; 20:562-9.

17. Alouini S, Uzan M, Meningaud JP, Herve C. Knowledge about contraception in women undergoing repeat voluntary abortions, and means of prevention. Eur J Obstet Gynecol Reprod Biol 2002; 104:43-8.

18. Forrest JD. Epidemiology of unintended pregnancy and contraceptive use. Am J Obstet Gynecol 1994; 170 (5 Pt 2):1485-9.

19. Carvalho MLO, Pirotta KCM, Schor N. Participação masculina na contracepção pela ótica feminina. Rev Saúde Pública 2001; 35:23-31.

20. Duarte GA, Alvarenga AT, Osis MJD, Faúndes A, Sousa MH. Participação masculina no uso de métodos contraceptivos. Cad Saúde Pública 2003; 19:207-16.

21. Jones RK, Darroch JE, Henshaw SK. Contraceptive use among U.S. women having abortions in 2000-2001. Perspect Sex Reprod Health 2002; 34:294-303.

22. Osis MJD, Faúndes A, Souza MH, Bailey P. Conseqüências do uso de métodos anticoncepcionais na vida das mulheres: o caso da laqueadura tubária. Cad Saúde Pública 1999; 15:521-32.

23. Associação Nacional de Empresas de Pesquisa. Critério de classificação econômica do Brasil. São Paulo: Associação Nacional de Empresas de Pesquisa; 2002.

24. Victora CG, Huttly SR, Fuchs SC, Olinto MT. The role of conceptual frameworks in epidemiological analysis: a hierarchical approach. Int J Epidemiol 1997; 26:224-7.

25. Dias-da-Costa JS, Facchini LA. Utilização de serviços ambulatoriais em Pelotas: onde a população consulta e com que freqüência. Rev Saúde Pública 1997; 31:360-9.

26. Nichols D, Woods ET, Gates DS, Sherman J. Sexual behavior, contraceptive practice, and reproductive health among Liberian adolescents. Stud Fam Plann 1987; 18:169-76.

27. Ezeh AC, Mboup G. Estimates and explanations of gender differentials in contraceptive prevalence rates. Stud Fam Plann 1997; 28:104-21.

28. Casterline JB, Sathar ZA, ul Haque M. Obstacles to contraceptive use in Pakistan: a study in Punjab. Stud Fam Plann 2001; 32:95-110.

Recebido em 01/Dez/2004

Versão final reapresentada em 19/Abr/2005

Aprovado em 09/Mai/2005 